

\title{
The semistable reduction problem for the space of morphisms on $\mathbb{P}^{n}$
}

\author{
Alon Levy
}

\begin{abstract}
We restate the semistable reduction theorem from geometric invariant theory in the context of spaces of morphisms from $\mathbb{P}^{n}$ to itself. For every complete curve $C$ downstairs, we get a $\mathbb{P}^{n}$-bundle on an abstract curve $D$ mapping finite-to-one onto $C$, whose trivializations correspond to not necessarily complete curves upstairs with morphisms corresponding to identifying each fiber with the morphism the point represents. Finding a trivial bundle is equivalent to finding a complete $D$ upstairs mapping finite-to-one onto $C$; we prove that in every space of morphisms, there exists a curve $C$ for which no such $D$ exists. In the case when $D$ exists, we bound the degree of the map from $D$ to $C$ in terms of $C$ for $C$ rational and contained in the stable space.
\end{abstract}

\section{Introduction and the statement of the problem}

The moduli spaces of dynamical systems on $\mathbb{P}^{n}$ are the spaces of morphisms, and more generally rational maps, defined by polynomials of degree $d$; the case we will study is $d>1$, in which case the morphisms are not automorphisms (that is, they do not have inverses that are morphisms). For each $n$ and $d$, we write each rational map $\varphi$ as $\left(\varphi_{0}: \cdots: \varphi_{n}\right)$, so that the space is parametrized by the coefficients of the monomials of each $\varphi_{i}$ and is naturally isomorphic to a large projective space, $\mathbb{P}^{N}$. By an elementary computation, $N=(n+1)\left(\begin{array}{c}n+d \\ d\end{array}\right)-1$. As we will not consider more than one of these moduli spaces at a time, there is no ambiguity in writing just $N$, without explicit dependence on $n$ and $d$. Thus, in the remainder of this paper, $N$ will invariably be used for the dimension of the moduli space of self-maps on $\mathbb{P}^{n}$ defined by polynomials of degree $d$.

Within the space of rational maps, the space of morphisms is an affine open subvariety, denoted $\operatorname{Hom}_{d}^{n}$. The group PGL $(n+1)$ acts on $\mathbb{P}^{N}$ by conjugation, corresponding to coordinate change, that is, $A$ maps $\varphi$ to $A \varphi A^{-1}$; this action preserves $\mathrm{Hom}_{d}^{n}$, since the property of being a morphism is independent of coordinate change.

MSC2010: primary 14L24; secondary 37P45, 37P55.

Keywords: semistable reduction, moduli space, dynamical system, GIT, geometric invariant theory. 
We study the quotient of the action using geometric invariant theory [Mumford and Fogarty 1982]. To do this, we need to replace PGL $(n+1)$ with $\operatorname{SL}(n+1)$, which projects onto PGL $(n+1)$ finite-to-one. Geometric invariant theory defines stable and semistable loci for the $\operatorname{SL}(n+1)$-action. To take the quotient, we need to remove the unstable locus, defined as the complement of the semistable locus. The quotient of $\operatorname{Hom}_{d}^{n}$ by $\operatorname{SL}(n+1)$ is denoted $\mathrm{M}_{d}^{n}$, and parametrizes morphisms on $\mathbb{P}^{n}$ up to coordinate change. The stable and semistable loci for the action of $\operatorname{SL}(n+1)$ on $\mathbb{P}^{N}$ are denoted by $\operatorname{Hom}_{d}^{n, s}$ and $\operatorname{Hom}_{d}^{n, s s}$, and their quotients are denoted by $\mathrm{M}_{d}^{n, s}$ and $\mathbf{M}_{d}^{n, s s}$.

It is a fact that every regular map is in the stable locus. More precisely, we have the following prior results [Silverman 1998; Petsche et al. 2009; Levy 2011]:

Theorem 1.1. $\operatorname{Hom}_{d}^{n, s}$ and $\operatorname{Hom}_{d}^{n, s s}$ are open subvarieties of $\mathbb{P}^{N}$ such that $\operatorname{Hom}_{d}^{n} \subsetneq$ $\operatorname{Hom}_{d}^{n, s} \subseteq \operatorname{Hom}_{d}^{n, s s} \subsetneq \mathbb{P}^{N}$. The middle containment is an equality if and only if $n=1$ and $d$ is even.

Theorem 1.2. The stabilizer group in $\operatorname{PGL}(n+1)$ of each element of $\mathrm{Hom}_{d}^{n}$ is finite and bounded in terms of $d$ and $n$.

$\mathrm{M}_{d}^{n, s s}$ is a proper variety, as it is the quotient of the largest semistable subspace of $\mathbb{P}^{N}$ for the action of $\operatorname{SL}(n+1)$. We make the following simplifying definition.

Definition 1.3. A rational map $\varphi \in \mathbb{P}^{N}$ is called semistable if it is in the semistable space $\operatorname{Hom}_{d}^{n, s s}$.

The semistable reduction theorem states the following, answering in the affirmative a conjecture for $\mathbb{P}^{1}$ in [Szpiro et al. 2010]:

Theorem 1.4. If $C$ is a complete curve with $K(C)$ its function field, and if $\varphi_{K(C)}$ is a semistable rational map on $\mathbb{P}_{K(C)}^{n}$, then there exists a curve $D$ mapping finite-to-one onto $C$ with a $\mathbb{P}^{n}$-bundle $\boldsymbol{P}(\mathscr{E})$ on $D$ with a self-map $\Phi$ such that:

(1) The restriction $\varphi_{x}$ of $\Phi$ to the fiber of each $x \in D$ is a semistable rational self-map.

(2) $\Phi$ is a semistable map over $K(D)$, and is equivalent to $\varphi_{K(D)}$ under coordinate change.

This is a classical result of geometric invariant theory; for one proof of a result that implies it, see [Zhang 1996]. We will include the proof in Section 2, along with other general facts about geometric invariant theory, including a description of the stable and semistable spaces $\operatorname{Hom}_{d}^{n, s}$ and $\operatorname{Hom}_{d}^{n, s s}$.

Theorem 1.4 leads to the natural question of which vector bundle classes can occur for each $C \subseteq \mathrm{M}_{d}^{n, s s}$, and more generally, for each choice of $n$ and $d$. One interesting subquestion is whether, for every $C$, we can choose the bundle to be trivial. Equivalently, given $C$, it asks whether we can find a proper $D \subseteq \operatorname{Hom}_{d}^{n, s s}$ 
that maps finite-to-one onto $C$. For most curves upstairs, the answer should be positive, by simple dimension counting: as demonstrated in [Silverman 1998] and [Levy 2011], the complement of $\operatorname{Hom}_{d}^{n, s s}$ has high codimension, equal to about half of $N$. However, it turns out that the answer is sometimes negative, and in fact, for every $n$ and $d$, we can find a $C$ with only nontrivial bundle classes. More precisely:

Theorem 1.5. For every $n$ and $d$, there exists a curve with no trivial bundle class satisfying semistable reduction.

Remark 1.6. An equivalent formulation for Theorem 1.5 is that for every $n$ and $d$, we can find a curve $C \subseteq \mathrm{M}_{d}^{n, s s}$ such that there does not exist a curve $D \subseteq \operatorname{Hom}_{d}^{n, s s}$ mapping onto $C$ under $\pi$.

Although most curves in $\operatorname{Hom}_{d}^{n, s s}$ can be completed, this does not imply that we can find a nontrivial bundle on an open dense set of the Chow variety of $\mathrm{M}_{d}^{n \text {,ss }}$. In fact, as we will see in Section 5, there exist components of the Chow variety of $\mathrm{M}_{d}^{n, s s}$ where, at least generically, a nontrivial bundle is required.

Our study of bundle classes now splits into two cases. In the case of curves satisfying semistable reduction with a trivial bundle, the reformulation of Remark 1.6, in its positive form, means that we can study $D$ directly as a curve in $\mathbb{P}^{N}$. We can bound the degree of the map from $D$ to $C$ in terms of the stabilizer groups that occur on $D$. More precisely:

Proposition 1.7. Let $X$ be a projective variety over an algebraically closed field with an action by a geometrically reductive linear algebraic group $G$. Using the terminology of geometric invariant theory, let $D$ be a complete curve in the stable space $X^{s}$ whose quotient by $G$ is a complete curve $C$; say the map from $D$ to $C$ has degree $m$. Suppose the stabilizer is generically finite of size $h$, and either $D$ or $C$ is normal. Then there exists a finite subgroup $S_{D} \subseteq G$, of order equal to $m h$, such that for all $x \in D$ and $g \in G, g x \in D$ if and only if $g \in S_{D}$.

Corollary 1.8. With the same notation and conditions as in Proposition 1.7, the map from $D$ to $C$ is ramified precisely at points $x \in D$ where the stabilizer group is larger than $h$, and intersects $S_{D}$ in a larger subgroup than in the generic case.

If the genus of $C$ is 0 , then the only way the map from $D$ to $C$ could have high degree is if it ramifies over many points; therefore, Corollary 1.8 forces the degree to be small, at least as long as $C$ is contained in the stable locus.

In the case of curves that only satisfy semistable reduction with a nontrivial bundle, we do not have a description purely in terms of coordinates. Instead, we will study which bundle classes can be attached to every curve $C$. The question of which bundles occur is an invariant of $C$; therefore, it is essentially an invariant that we can use to study the scheme $\operatorname{Hom}\left(C, \mathrm{M}_{d}^{n, s s}\right)$. In the sequel, we will study the scheme using the bundle class set and height invariants. 
For the study of which nontrivial bundle classes can occur, first observe that fixing a $D$ for which a bundle exists, we can apply the reformulation of Theorem 2.11 to obtain a unique extension of $\varphi$ locally. This can be done at every point, so it is true globally, so we have:

Proposition 1.9. Using the notation of Theorem 1.4, the bundle class $\boldsymbol{P}(\mathscr{E})$ depends only on $D$ and its trivialization $U_{i}, U_{i} \hookrightarrow \operatorname{Hom}_{d}^{n, s s}$.

Note that the bundle class does not necessarily depend only on $D$, regarded as an abstract curve with a map to $C$. The reason is that a point of $D$ may not be stable, which means it may correspond to one of several different orbits, whose closures intersect. However, there are only finitely many orbits corresponding to each point, so the bundle class depends on $D$ up to a finite amount; if $C$ happens to be contained in the stable locus, then it depends only on $D$.

Thus we can study which bundle classes occur for a given $C$. We will content ourselves with rational curves, for which there is a relatively easy description of all projective bundles. Recall that every vector bundle over $\mathbb{P}^{1}$ splits as a direct sum of line bundles, and that the bundle $\bigoplus_{i} O\left(m_{i}\right)$ is projectively equivalent to $\bigoplus_{i} \mathcal{O}\left(l+m_{i}\right)$ for all $l \in \mathbb{Z}$. In other words, a $\mathbb{P}^{n}$-bundle over $\mathbb{P}^{1}$ can be written as $\mathscr{O} \oplus \mathcal{O}\left(m_{1}\right) \oplus \cdots \oplus \mathcal{O}\left(m_{n}\right)$; if the $m_{i}$ 's are in nondecreasing order, then the expression uniquely determines the bundle's class. We will show that:

Proposition 1.10. There exists a curve $C$ for which multiple nonisomorphic bundle classes can occur. In fact, suppose $C$ is isomorphic to $\mathbb{P}^{1}$, and there exists $U \subseteq$ $\operatorname{Hom}_{d}^{n \text {,ss }}$ mapping finite-to-one into $C$ such that $U$ is a projective curve minus a point. Then there are always infinitely many possible classes: if the class of $U$ is thought of as splitting as $\boldsymbol{P}(\mathscr{E})=\mathbb{O} \oplus \mathcal{O}\left(m_{1}\right) \oplus \cdots \oplus \mathcal{O}\left(m_{n}\right)$, where $m_{i} \in \mathbb{N}$, then for every integer l the class $\mathbb{O} \oplus \mathbb{O}\left(l_{1}\right) \oplus \cdots \oplus \mathcal{O}\left(l m_{n}\right)$ also occurs.

Proposition 1.10 frustrated our initial attempt to obtain an easy classification of bundles based on curves. However, it raises multiple interesting questions instead. First, the construction uses a rational $D$ mapping finite-to-one onto $C$, and going to higher $m$ involves raising the degree of the map $D \rightarrow C$. It may turn out that bounding the degree bounds the bundle class; we conjecture that if we fix the degree of the map, then we obtain only finitely many bundle classes. Furthermore, in analogy with the consequences of Corollary 1.8 , we should conversely be able to bound the degree of the map in terms of $C$ and the bundle class, at least for rational C.

Second, it is nontrivial to find the minimal $m_{i}$ 's for which a bundle splitting as $\mathcal{O} \oplus \mathscr{O}\left(m_{1}\right) \oplus \cdots \oplus \mathcal{O}\left(m_{n}\right)$ would satisfy semistable reduction; the case of $n=1$ could be stated particularly simply, as the question would be about the minimal $\mathrm{m}$ for which $\mathbb{O} \oplus \mathbb{O}(m)$ occurs. 
In Sections 3 and 4 we will illustrate Theorem 1.5: in Section 3 we will give some examples and compute the bundle classes that occur, proving Proposition 1.10 on the way, while in Section 4 we will prove Theorem 1.5. In Section 5 we will focus on the trivial bundle case, proving Proposition 1.7 and defining the height function, which will impose constraints on which curves admit a trivial bundle; this will allow us to obtain a large family of curves $C$ in $\mathrm{M}_{2}^{s s}$ with no trivial bundle.

\section{A description of the stable and semistable spaces}

Unless another reference is given, the general geometric invariant theory results given in this section are all from [Mumford and Fogarty 1982].

Recall that when a geometrically reductive linear algebraic group $G$ has a linear action on a projectivized vector space $\mathbb{P}(V)$, we have:

Definition 2.1. A point $x \in V$ is called semistable (resp. stable) if any of the following equivalent conditions hold:

(1) There exists a $G$-invariant homogeneous section $s$ such that $s(x) \neq 0$ (resp. same condition, and the action of $G$ on $x$ is closed).

(2) The closure of $G \cdot x$ does not contain 0 (resp. $G \cdot x$ is closed).

(3) Every one-parameter subgroup $T$ acts on $x$ with both nonnegative and nonpositive weights (resp. negative and positive weights).

Remark 2.2. The last condition in the definition is equivalent to having nonpositive (resp. negative) weights. This is because if we can find a subgroup acting with only negative weights, then we can take its inverse and obtain only positive weights.

Observe that for every nonzero scalar $k, x$ is stable (resp. semistable) if and only if $k x$ is. So the same definitions of stability and semistability hold for points of $\mathbb{P}(V)$. The definitions also descend to every $G$-invariant projective variety $X \subseteq \mathbb{P}(V)$; in fact, in [Mumford and Fogarty 1982] they are defined for $X$ in terms of a $G$-equivariant line bundle $L$. When $L$ is ample, as in the case of the space under discussion in this paper, this reduces to the above definition.

The importance of stability is captured in the following results:

Proposition 2.3. The space of all stable points, $X^{s}$, and the space of all semistable points, $X^{s s}$, are both open and $G$-invariant.

Theorem 2.4. There exists a quotient $Y=X^{s s} / / G$, called a good categorical quotient (in the category of separated schemes), with a natural map $\pi: X \rightarrow Y$, satisfying the following properties:

(1) $\pi$ is a $G$-equivariant map, where $G$ acts on $Y$ trivially.

(2) Every G-equivariant map $X \rightarrow W$, where $G$ acts on $W$ trivially, factors through $\pi$. 
(3) $\pi$ is an open submersion.

(4) $\pi\left(x_{1}\right)=\pi\left(x_{2}\right)$ if and only if the closures of $G \cdot x_{1}$ and $G \cdot x_{2}$ intersect.

(5) For every open $U \subseteq Y, O_{U}=\mathbb{O}\left(\pi^{-1}(U)\right)^{G}$.

In addition, $Y$ is proper.

Theorem 2.5. There exists a quotient $Z=X^{s} / / G$, called a good geometric quotient, with a natural map $\pi: X \rightarrow Z$ satisfying all enumerated conditions of a good categorial quotient, as well as the following:

(1) $\pi\left(x_{1}\right)=\pi\left(x_{2}\right)$ if and only if $G \cdot x_{1}=G \cdot x_{2}$.

(2) $Z$ is naturally an open subset of $X^{s s} / / G$.

Theorem 2.6. On $X^{s}$, the dimension of the stabilizer group $\operatorname{Stab}_{G}(x)$ is constant.

Returning to our case of self-maps of $\mathbb{P}^{n}$, we write the stable and semistable spaces for the conjugation action as $\operatorname{Hom}_{d}^{n, s}$ and $\operatorname{Hom}_{d}^{n, s s}$. This involves a fair amount of abuse of notation, since those two spaces are open subvarieties of $\mathbb{P}^{N}$ and in fact properly contain $\operatorname{Hom}_{d}^{n}$, which consists only of regular maps.

In [Levy 2011] we proved the fact that $\operatorname{Hom}_{d}^{n} \subsetneq \operatorname{Hom}_{d}^{n, s}$ by describing $\operatorname{Hom}_{d}^{n, s}$ and $\operatorname{Hom}_{d}^{n, s s}$ more or less explicitly. We will recapitulate the results, which are very technical but help us answer the question of when we can obtain a trivial bundle class in the semistable reduction problem and when we cannot.

We use the Hilbert-Mumford criterion, the last condition in Definition 2.1. In more explicit terms, the criterion for semistability (resp. stability) states that for every one-parameter subgroup $T \leq \mathrm{SL}(n+1)$, the action of $T$ on $\varphi$ can be diagonalized with eigenvalues $t^{a_{I}}$ and at least one $a_{I}$ is nonpositive (resp. negative). Now, assume by conjugation that this one-parameter subgroup is in fact diagonal, with diagonal entries $t^{a_{0}}, \ldots, t^{a_{n}}$, and that $a_{0} \geq \cdots \geq a_{n}$; we may also assume that the $a_{i}$ 's are coprime, as dividing throughout by a common factor would not change the underlying group. Note also that $a_{0}+\cdots+a_{n}=0$. Our task is made easy by the fact that our standard coordinates for $\mathbb{A}^{N+1}$ are the monomials, on which $T$ already acts diagonally. Throughout this analysis, we fix $\boldsymbol{a}=\left(a_{0}, \ldots, a_{n}\right)$, and similarly for $\boldsymbol{x}$ and $\boldsymbol{d}$.

Now, $T$ acts on the $x_{0}^{d_{0}} \ldots x_{n}^{d_{n}}$ monomial of the $i$-th polynomial, $\varphi_{i}$, with weight $a_{i}-\boldsymbol{a} \cdot \boldsymbol{d}$. A map $\varphi \in \mathbb{P}^{N}$ is unstable (resp. not stable) if and only if, after conjugation, there exists a choice of $a_{i}$ 's such that whenever the $\mathbf{x}^{\mathbf{d}}$-coefficient of $\varphi_{i}$ satisfies $\boldsymbol{a} \cdot \boldsymbol{d} \leq a_{i}$ (resp. $<$ ), it is equal to zero.

Remark 2.7. While in principle there are infinitely many possible $T$ 's, parametrized by a hyperplane in $\mathbb{P}^{n}(\mathbb{Q})$, in practice there are up to conjugation only finitely many. This is because each diagonal $T$ imposes conditions of the form "the $\mathbf{x}^{\mathbf{d}}$-coefficient of $\varphi_{i}$ is zero," and there are only finitely many such conditions. Thus the stable and semistable spaces are indeed open in $\mathbb{P}^{N}$. 
Remark 2.8. The conjugation conditions we have chosen for $T$ are such that the conditions they impose for $\varphi$ to be unstable (or merely not stable) are the most stringent on $\varphi_{n}$ and least stringent on $\varphi_{0}$, and are the most stringent on monomials with high $x_{0}$-degrees and least stringent on monomials with high $x_{n}$-degrees.

If $n=1$, we have a simpler description:

Theorem 2.9 [Silverman 1998]. $\varphi \in \mathbb{P}^{N}$ is unstable (resp. not stable) if and only if it is equivalent under coordinate change to a map

$$
x \mapsto \frac{a_{0} x^{d}+\cdots+a_{d} y^{d}}{b_{0} x^{d}+\cdots+b_{d} y^{d}}
$$

such that:

(1) $a_{i}=0$ for all $i \leq(d-1) / 2$ (resp. $\left.<\right)$.

(2) $b_{i}=0$ for all $i \leq(d+1) / 2$ (resp. $<$ ).

The description for $n=1$ can be thought of as giving a dynamical criterion for stability and semistability. A point $\varphi \in \mathbb{P}^{N}$ is unstable if there exists a point $x \in \mathbb{P}^{1}$ where $\varphi$ has a bad point of degree more than $(d+1) / 2$, or $\varphi$ has a bad point of degree more than $(d-1) / 2$ where it in addition has a fixed point. Following Rahul Pandharipande's unpublished reinterpretation of [Silverman 1998], we define "bad point" as a vertical component of the graph $\Gamma_{\varphi} \subseteq \mathbb{P}^{1} \times \mathbb{P}^{1}$, and "fixed point" as a fixed point of the unique nonvertical component of $\Gamma_{\varphi}$. When $n=1, d=2$, this condition reduces to having a fixed point at a bad point, or alternatively, a repeated bad point.

The conditions for higher $n$ are not as geometric. However, if we interpret fixed points liberally enough, there are still strong parallels with the $n=1$ case. One can show that the unstable space for $n=2$ and $d=2$ consists of two irreducible components, which roughly generalize the $n=1, d=2$ condition of having a fixed point at a bad point; in this case, one needs to define a limit of the value of $\varphi(x)$ as $x$ approaches the bad point, though this limit can be defined purely in terms of degrees of polynomials, without needing to resort to a specific metric on the base field.

Finally, let us prove semistable reduction. Let us restate Theorem 1.4:

Theorem 2.10. If $C$ is a complete curve with $K(C)$ its function field, and if $\varphi_{K(C)}$ is a semistable rational map on $\mathbb{P}_{K(C)}^{n}$, then there exists a curve $D$ mapping finiteto-one onto $C$ with a $\mathbb{P}^{n}$-bundle $\boldsymbol{P}(\mathscr{E})$ on $D$ with a self-map $\Phi$ such that:

(1) The restriction $\varphi_{x}$ of $\Phi$ to the fiber of each $x \in D$ is a semistable rational self-map.

(2) $\Phi$ is a semistable map over $K(D)$, and is equivalent to $\varphi_{K(D)}$ under coordinate change. 
Semistable reduction can be thought of as extending a rational map defined over a field $K$ to a rational map defined over a discrete valuation ring $R$ whose fraction field is $K$, in a way that is not too degenerate. The reason a discrete valuation ring suffices is that once we know we can extend to a discrete valuation ring, we can extend to some larger integral domain. In other words, it suffices to show the following, more general statement:

Theorem 2.11. Let $G$ be a geometrically reductive group acting on a projective variety $X$ whose stable and semistable spaces are $X^{s}$ and $X^{\text {ss }}$, respectively. Let $R$ be a discrete valuation ring with fraction field $K$, and let $x_{K} \in X_{K}^{s}$. Then for some finite extension $K^{\prime}$ of $K$, with $R^{\prime}$ the integral closure of $R$ in $K^{\prime}, x_{K}$ has an integral model over $R^{\prime}$ with semistable reduction modulo the maximal ideal. In other words, we can find some $A \in G(\bar{K})$ such that $A \cdot x_{K}$ has semistable reduction. If $x_{K} \in X_{K}^{s s}$, then the same result is true, except that $x_{R^{\prime}}$ could be an integral model for some $x_{K^{\prime}}^{\prime}$ mapping to the same point of $X^{s s} / / G$ such that $x_{K^{\prime}}^{\prime} \notin G \cdot x_{K}$.

Proof. We follow the method used in [Zhang 1996]. Let $C$ be the Zariski closure of $x_{K}$ in $X_{R}^{s s} / / G$, and reduce it modulo the maximal ideal to obtain $x_{k}$, where $k$ is the residue field of $R$. Observe that $C$ is a one-dimensional subscheme of $X_{R}^{s s} / / G$ and is isomorphic to $\operatorname{Spec} R$, and is as a result connected. Since $G$ is connected, the preimage $\pi^{-1}(C)$ is also connected: when $x_{K}$ is stable, this follows from the fact that $\pi^{-1}(C)$ is the Zariski closure of $G \cdot x_{K}$ in $X^{s s}$, and even when it is not, $\pi^{-1}(C)$ is the union of connected orbits whose closures intersect. Further, since $\pi^{-1}(C)$ surjects onto $C$, we can find an integral one-dimensional subscheme mapping surjectively to $C$. This subscheme necessarily maps finite-to-one onto $C$ by dimension counting, so it is isomorphic to some finite extension ring $R^{\prime}$, giving us $K^{\prime}$ as its fraction field.

Remark 2.12. Theorem 2.11 can also be proven in a much more explicit way, producing for each $\varphi_{K} \in \operatorname{Hom}_{d}^{n, s s}$ a sequence of $A$ 's conjugating it to a model with semistable reduction.

Remark 2.13. Szpiro et al. [2010] study semistable reduction for the moduli space of self-maps of $\mathbb{P}^{1}$ and raise a conjecture that Theorems 1.4 and 2.11 answer in the affirmative.

\section{Examples of nontrivial bundles}

In the case $n=1$, we follow [Silverman 1998] and write $\mathrm{Rat}_{d}$ for $\operatorname{Hom}_{d}^{1}$ and $\mathrm{M}_{d}$ for $M_{d}^{1}$. The space Rat 2 and its quotient $\mathrm{M}_{2}$ have been analyzed with more success than the larger spaces, yielding the following prior structure result: 
Theorem 3.1 [Milnor 2006; Silverman 1998]. $\mathrm{M}_{2}=\mathbb{A}^{2} ; \mathrm{M}_{2}^{s}=\mathrm{M}_{2}^{s s}=\mathbb{P}^{2}$. The first two elementary symmetric polynomials in the multipliers of the fixed points realize both isomorphisms.

Recall that within $\mathbb{P}^{N}=\mathbb{P}^{5}$, a map $\left(a_{0} x^{2}+a_{1} x y+a_{2} y^{2}\right) /\left(b_{0} x^{2}+b_{1} x y+b_{2} y^{2}\right)$ is unstable if and only if it is in the closure of the PGL(2)-orbit of the subvariety $a_{0}=b_{0}=b_{1}=0$. In other words, it is unstable if and only if there the map is degenerate and has a double bad point, or a fixed point at a bad point.

Definition 3.2. A map on $\mathbb{P}^{1}$ is a polynomial if and only if there exists a totally invariant fixed point. Taking such a point to infinity turns the map into a polynomial in the ordinary sense. In Rat ${ }_{d}$, or generally in $\mathbb{P}^{N}=\mathbb{P}^{2 d+1}$, a map is polynomial if and only if it is in the closure of the PGL(2)-orbit of the subvariety defined by zeros in all coefficients in the denominator except the $y^{d}$-coefficient.

Remark 3.3. A totally invariant fixed point is not necessarily a totally fixed point. A totally invariant fixed point is one that is totally ramified. A totally fixed point is the root of the fixed point polynomial when it is unique, that is, when the polynomial is a power of a linear term. In fact by an easy computation, a map has a totally invariant, totally fixed point $x$ if and only if it is degenerate linear with bad point of multiplicity $d-1$ at $x$, in which case it is necessarily unstable.

The polynomial maps define a curve in $\mathbf{M}_{2}^{s s}$; we will show:

Proposition 3.4. The polynomial curve in $\mathrm{M}_{2}^{s s}$ only satisfies semistable reduction with nontrivial bundles.

Proof. First, note that in $\mathbb{P}^{5}$, the polynomial maps are those that can be conjugated to the form $\left(a_{0} x^{2}+a_{1} x y+a_{2} y^{2}\right) / b_{2} y^{2}$, in which case the totally invariant fixed point is $\infty=(1: 0)$. We will call the polynomial map locus $X$. If $a_{0}=0$, then the map is unstable; we will show that every curve in $X$ contains a map for which $a_{0}=0$. Clearly, the set of all maps with a given totally invariant fixed point is isomorphic to $\mathbb{P}^{3}$, and the unstable locus within it is isomorphic to $\mathbb{P}^{2}$ as a linear subvariety, so for there to be any hope of a trivial bundle, a curve in $X$ cannot lie entirely over one totally invariant point.

Now, the fixed point equation for a map of the form $f / g$ is $f y-g x=0$; the homogeneous roots of this equation are the fixed points, with the correct multiplicities. For our purposes, when the totally invariant point is $\infty$, the fixed point equation is $a_{0} x^{2} y+\left(a_{1}-b_{2}\right) x y^{2}+a_{2} y^{3}=0$. We get that $a_{0}=0$ if and only if the totally invariant point is a repeated root of the fixed point equation.

There exists a map from $X$ to $\mathbb{P}^{1} \times \mathbb{P}^{2}$, mapping $\varphi$ to its totally invariant point in $\mathbb{P}^{1}$, and to the two elementary symmetric polynomials in the two other fixed points in $\mathbb{P}^{2}$. Write $(x: y)$ for the image in $\mathbb{P}^{1}$ and $(a: b: c)$ for the image in $\mathbb{P}^{2}$. Now $(x: y)$ is a repeated root if $a x^{2}+b x y+c y^{2}=0$. The equation defines an 
ample divisor, so every curve in $\mathbb{P}^{1} \times \mathbb{P}^{2}$ will meet it. Finally, a curve in $X$ maps either to a single point in $\mathbb{P}^{1} \times \mathbb{P}^{2}$, in which case it must contain points with $a_{0}=0$ as above, or to a curve, in which case it intersects the divisor $a x^{2}+b x y+c y^{2}=0$. In both cases, the curve contains unstable points. Thus there is no global semistable curve $D$ in $\mathrm{Rat}_{2}^{s s}$ mapping down to $C$.

Note that in the above proof, maps conjugate to $x^{2}$ have two totally invariant points, so a priori the map from $X$ to $\mathbb{P}^{1} \times \mathbb{P}^{2}$ is not well-defined at them. However, for any curve $D$ in $X$, there is a well-defined completion of this map, whose value at $x^{2}$ on the $\mathbb{P}^{1}$ factor is one of the two totally invariant points. Thus this complication does not invalidate the above proof.

Let us now compute the vector bundle classes that do occur for the polynomial curve. We work with the description $x^{2}+c$, which yields an affine curve that maps one-to-one into $C$, missing only the point at infinity, which is conjugate to $\left(x^{2}-x\right) / 0$. To hit the point at infinity, we choose the alternative parametrization $c x^{2}-c x+1$, which, when $c=\infty$, corresponds to the unique (up to conjugation) semistable degenerate constant map. For any $c$, this map is conjugate to $x^{2}-c x+c$ and thence $x^{2}+c / 2-c^{2} / 4$, using the transition function $\left[c,-\frac{1}{2} ; 0,1\right]$. Thus the bundle splits as $\mathscr{O} \oplus \mathbb{O}(1)$.

This bundle depends on the choice of $D$. In fact, if we choose another parametrization for $D$, for example $c^{2} x^{2}-c^{2} x+1$, then the transition function is $\left[c^{2},-\frac{1}{2} ; 0,1\right]$, which leads to the bundle $\mathscr{O} \oplus \mathcal{O}(2)$. This is not equivalent to $\mathscr{O} \oplus \mathcal{O}(1)$. This then leads to the question of which classes of bundles can occur over each $C$. In the example we have just done, the answer is every nontrivial class: for every positive integer $m$, we can use $c^{m} x^{2}-c^{m} x+1$ as a parametrization, leading to $\mathcal{O} \oplus \mathbb{O}(m)$, which exhausts all nontrivial projective bundle classes.

Recall the result of Proposition 1.10:

Proposition 3.5. Suppose $C$ is isomorphic to $\mathbb{P}^{1}$, and there exists $U \subseteq \operatorname{Hom}_{d}^{n, s s}$ mapping finite-to-one into $C$ such that $U$ is a projective curve minus a point. Then there are always infinitely many possible classes: if the class of $U$ is thought of as splitting as $\boldsymbol{P}(\mathscr{E})=\mathscr{O} \oplus \mathcal{O}\left(m_{1}\right) \oplus \cdots \oplus \mathcal{O}\left(m_{n}\right)$, where $m_{i} \in \mathbb{N}$, then for every integer $l$, the class $\mathrm{O} \oplus \mathrm{O}\left(\operatorname{lm}_{1}\right) \oplus \cdots \oplus \mathcal{O}\left(\operatorname{lm}_{n}\right)$ also occurs.

Proof. Imitating the analysis of the polynomial curve above, we can parametrize $C$ by one variable, say $c$, and choose coordinates such that the sole bad point in the closure of $U$ corresponds to $c=\infty$. Now, we can by assumption find a piece $U^{\prime}$ above the infinite point with a transition function determining the vector bundle $\mathcal{O} \oplus \mathcal{O}\left(m_{1}\right) \oplus \cdots \oplus \mathcal{O}\left(m_{n}\right)$. Now let $V$ be the composition of $U^{\prime}$ with the map $c \mapsto c^{l}$. Then $U$ and $V$ determine a vector bundle satisfying semistable reduction, of class $\mathcal{O} \oplus \mathcal{O}\left(l m_{1}\right) \oplus \cdots \oplus \mathcal{O}\left(l m_{n}\right)$, as required.

The example in Proposition 3.4, of polynomial maps, is equivalent to a multiplier 
condition. When $d=2$, a map is polynomial if and only if it has a superattracting fixed point, that is, one whose multiplier is zero; see the description in the first chapter of [Silverman 2007]. One can imitate the proof that semistable reduction does not hold for a more general curve, defined by the condition that there exists a fixed point of multiplier $t \neq 1$. In that case, the condition $b_{1}=0$ is replaced by $b_{1}=t a_{0}$, and the point is a repeated root of the fixed point equation if and only if $a_{0}=b_{1}$, in which case we clearly have $a_{0}=b_{1}=0$ and the point is unstable.

When the multiplier is 1 , the fixed point in question is automatically a repeated root, with $b_{1}=a_{0}$. The condition that the point be the only fixed point corresponds to $b_{2}=a_{1}$, which by itself does not imply that the map fails to be a morphism, let alone that it is unstable.

Instead, the condition that gives us $b_{1}=a_{0}=0$ is the condition that the fixed point be totally invariant. Specifically, the fixed point's two preimages are itself and one more point; when the fixed point is $\infty$, the extra point is $-b_{2} / b_{1}$. Now we can map $X$ to $\mathbb{P}^{1} \times \mathbb{P}^{1}$, where the first coordinate is the fixed point and the second is its preimage. This map is well-defined on all of $X$ because only one point can be a double root of a cubic. Now the diagonal is ample in $\mathbb{P}^{1} \times \mathbb{P}^{1}$, so the only way a curve $D$ can avoid it is by mapping to a single point; but in that case, $D$ lies in a fixed variety isomorphic to $\mathbb{P}^{3}$ where the unstable locus is $\mathbb{P}^{2}$, so it will intersect the unstable locus.

The fact that any condition of the form "there exists a fixed point of multiplier $t$ " induces a curve for which semistable reduction requires a nontrivial bundle means that there is no hope of enlarging the semistable space in a way that ensures we always have a trivial bundle. We really do need to think of semistable reduction as encompassing nontrivial bundle classes as well as trivial ones.

Specifically: it is trivial to show that the closure of the polynomial locus in Rat 2 includes all the unstable points (fix $\infty$ to be the totally invariant point and let $a_{0}$ go to zero). At least some of those unstable points will also arise as closures of other multiplier- $t$ conditions. However, different multiplier- $t$ conditions limit to different points in $\mathrm{M}_{2}^{s s} \backslash \mathrm{M}_{2}$.

\section{The general case}

So far we have talked about nontrivial classes in $\mathrm{M}_{2}$. But we have a stronger result, restating Theorem 1.5:

Theorem 4.1. For all $n$ and $d$, over any base field, there exists a curve with no trivial bundle class satisfying semistable reduction.

Proof. In all cases, we will focus on polynomial maps, which we will define to be maps that are $\operatorname{PGL}(n+1)$-conjugate to maps for which the last polynomial $\varphi_{n}$ has zero coefficients in every monomial except possibly $x_{n}^{d}$. 
Lemma 4.2. The set of polynomial maps, defined above, is closed in $\overline{\operatorname{Hom}_{d}^{n}}=\mathbb{P}^{N}$.

Proof. Clearly, the set of polynomial maps with respect to a particular hyperplane for example, $x_{n}=0$ - is closed. Now, for each hyperplane $a_{0} x_{0}+\cdots+a_{n} x_{n}=0$, we can check by conjugation to see that the condition that the map be polynomial corresponds to the condition that $a_{0} \varphi_{0}+\cdots+a_{n} \varphi_{n}=c\left(a_{0} x_{0}+\cdots+a_{n} x_{n}\right)^{d}$, where $c$ may be zero. As $\mathbb{P}^{n}$ is proper, it suffices to show that the condition " $\varphi$ is polynomial with respect to $a_{0} x_{0}+\cdots+a_{n} x_{n}=0$ " is closed in $\left(\mathbb{P}^{n}\right)^{*} \times \mathbb{P}^{N}$.

Now, we may construct a rational function $f$ from $\left(\mathbb{P}^{n}\right)^{*} \times \mathbb{P}^{N}$ to $\operatorname{Sym}^{d}\left(\mathbb{P}^{n}\right) \times$ $\operatorname{Sym}^{d}\left(\mathbb{P}^{n}\right)$ by $\left(\left(a_{0} x_{0}+\ldots+a_{n} x_{n}\right), \varphi\right) \mapsto\left(\left(a_{0} x_{0}+\cdots+a_{n} x_{n}\right)^{d}, a_{0} \varphi_{0}+\cdots+a_{n} \varphi_{n}\right)$. The map $\varphi$ is polynomial with respect to $a_{0} x_{0}+\cdots+a_{n} x_{n}=0$ if and only if $f$ is ill-defined at $\left(\left(a_{0} x_{0}+\cdots+a_{n} x_{n}\right), \varphi\right)$ or $f\left(\left(a_{0} x_{0}+\cdots+a_{n} x_{n}\right), \varphi\right) \in \Delta$, the diagonal subvariety. The ill-defined locus of $f$ is closed, and the preimage of $\Delta$ is closed in the well-defined locus.

In fact, the condition of $\varphi$ being polynomial with respect to $r$ distinct hyperplanes in general position, where $r$ is a fixed integer - in other words, the condition that $\varphi$ be conjugate to a map for which $\varphi_{i}=c_{i} x_{i}^{d}$ for all $i>d-r$-is more or less closed as well. It is not closed, but a sufficiently good condition is closed. Namely:

Lemma 4.3. For each $1 \leq i \leq n$, consider the PGL $(n+1)$-orbit of the space of maps in which, for each $j \geq i, \varphi_{j}$ has zero coefficients in every monomial containing any term $x_{k}$ with $k<j$. This orbit is closed in $\mathbb{P}^{N}$.

Proof. Observe that the above-defined space of maps consists of maps that are polynomial with respect to $x_{n}=0$, such that the induced map on the totally invariant hyperplane $x_{n}=0$ is polynomial with respect to $x_{n-1}=0$, and so on until we reach the induced map on the totally invariant subspace $x_{i+1}=\cdots=x_{n}=0$.

Now we use descending induction. Lemma 4.2 is the base case, when $i=n$. Now suppose it is true down to $i$. Then for $i-1$, the condition of having no nonzero $x_{k}$ term in $\varphi_{i-1}$ with $k<i-1$ is equivalent to the condition that the induced map on the totally invariant subspace $x_{i}=x_{i+1}=\ldots=x_{n}=0$ be polynomial; this condition is closed in the space of all maps that are polynomial down to $x_{i}$, which we assume closed by the induction hypothesis.

Definition 4.4. We call maps of the form in Lemma 4.3 polynomial with respect to $\boldsymbol{B}$, where $B$ is the Borel subgroup preserving the ordered basis of conditions. In the case above, $B$ is the upper triangular matrices.

We need one final result to make computations easier:

Lemma 4.5. Let $X$ be a curve of polynomial maps, all with respect to a Borel subgroup $B$, and let $\varphi$ be a semistable map in $\overline{\operatorname{PGL}(n+1) \cdot X}$. Then $\varphi \in \overline{B \cdot X}$. 
Proof. Let $C$ be the closure of the image of $X$ in $\mathrm{M}_{d}^{n, s s}$. By semistable reduction, there exists some affine curve $Y \ni \varphi$ mapping finite-to-one to $C$, that is, dominantly. We need to find some open $Z \subseteq Y$ containing $\varphi$ and some $f: Z \rightarrow \operatorname{PGL}(n+1)$ such that $f(\varphi)$ is the identity matrix, and $Z^{\prime}=\{(f(z) \cdot z)\}$ consists of maps which are polynomial with respect to $B$. Such a map necessarily exists: we have a map $h$ from $Y$ to the flag variety of $\mathbb{P}^{n}$ sending each $y$ to the subgroup with respect to which it is polynomial (possibly involving some choice if generically $y$ is polynomial with respect to more than one flag), which then lifts to $G$, possibly after deleting finitely many points. Generically, a point of $X$ maps to a point of $C$ that is in the image of $Z$; therefore, picking the correct points in $X$, we get that $\varphi \in \overline{B \cdot X}$.

With the above lemmas, let us now prove Theorem 4.1 for $n=1$, which is slightly easier than the higher- $n$ case, where the more complicated Lemma 4.3 is required. We will use the family $x^{d}+c$, where $c \in \mathbb{A}^{1}$. In projective notation, this is $\left(a_{0} x^{d}+a_{d} y^{d}\right) / b_{d} y^{d}$, which is a one-dimensional family modulo conjugation.

Lemma 4.6. Let $V$ be the closure of the PGL(2)-orbit of the family

$$
\frac{a_{0} x^{d}+a_{d} y^{d}}{b_{d} y^{d}}
$$

in $\mathbb{P}^{N}$. Then:

(1) In characteristic 0 or $p \nmid d$, every $\varphi \in V$ is actually in the PGL(2)-orbit of the family, or else it is a degenerate linear map, conjugate to

$$
\frac{a_{d-1} x y^{d-1}+a_{d} y^{d}}{b_{d} y^{d}} .
$$

(2) In characteristic $p \mid d$, with $p^{m} \| d$ and $p^{m} \neq d$, every $\varphi \in V$ is in the PGL(2)orbit of the family or is a degenerate map conjugate to

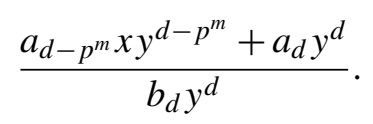

(3) In characteristic $p$ with $d=p^{m}$, set $V$ to be the closure of the orbit of the family $\left(a_{0} x^{d}+a_{d-1} x y^{d-1}\right) / b_{d} y^{d}$; then every $\varphi \in V$ is actually in the orbit of the family, or else it is a degenerate linear map, conjugate to

$$
\frac{a_{d-1} x y^{d-1}+a_{d} y^{d}}{b_{d} y^{d}}
$$

and furthermore, $a_{d-1}=b_{d}$.

Proof. Observe that the first two cases are really the same: case (2) is reduced to case (1) viewed as a degree- $\left(d / p^{m}\right)$ map in $\left(x^{p^{m}}: y^{p^{m}}\right)$. So it suffices to prove case (1) to prove (2); we will start with the family $\left(a_{0} x^{d}+a_{d} y^{d}\right) / b_{d} y^{d}$ and see 
what algebraic equations its orbit satisfies. As polynomials are closed in $\overline{\mathrm{Rat}_{d}}$, every point in the closure of the orbit is a polynomial. We may further assume it is polynomial with respect to $y=0$; therefore, by Lemma 4.5 , it suffices to look at the action of upper triangular matrices. Further, the condition of being within the family $\left(a_{0} x^{d}+a_{d} y^{d}\right) / b_{d} y^{d}$ is stabilized by diagonal matrices; therefore, it suffices to look at the action of matrices of the form $[1, t ; 0,1]$.

Now, the conjugation action of $[1, t ; 0,1]$ fixes $b_{d} y^{d}$ and maps $a_{0} x^{d}+a_{d} y^{d}$ to $a_{0}(x-t y)^{d}+\left(a_{d}+t b_{d}\right) y^{d}$. Clearly, there is no hope of obtaining any condition on $b_{d}$ or $a_{d}$. Now, the conditions on the terms $a_{0}, \ldots, a_{d-1}$ are that for some $t$, they fit into the pattern $a_{0}\left(x^{d}-d t x^{d-1} y+\cdots \pm d t^{d-1} x y^{d-1}\right)$, that is, $a_{i}=(-t)^{i}\left(\begin{array}{c}d \\ i\end{array}\right) a_{0}$. To remove the dependence on $t$, note that when $i+j=k+l$, we have $\left(\begin{array}{l}d \\ i\end{array}\right)\left(\begin{array}{l}d \\ j\end{array}\right) a_{i} a_{j}=\left(\begin{array}{l}d \\ k\end{array}\right)\left(\begin{array}{l}d \\ l\end{array}\right) a_{k} a_{l}$, as long as $i, j, k, l<d$.

Let us now look at what those conditions imply. Setting $j=i, k=i-1, l=i+1$, we get conditions of the form $\left(\begin{array}{c}d \\ i\end{array}\right)^{2} a_{i}^{2}=\left(\begin{array}{c}d \\ i-1\end{array}\right)\left(\begin{array}{c}d \\ i+1\end{array}\right) a_{i-1} a_{i+1}$, whenever $i+1<d$. If $a_{0} \neq 0$, then the value of $a_{1}$ uniquely determines the value of $a_{2}$ by the condition with $i=1$; the value of $a_{2}$ uniquely determines $a_{3}$ by the condition with $i=2$; and so on, until we uniquely determine $a_{d-1}$. In this case, choosing $t=-a_{1} / d a_{0}$ will conjugate this map back to the family $\left(a_{0} x^{d}+a_{d} y^{d}\right) / b_{d} y^{d}$. If $a_{0}=0$, then the equation with $i=1$ will imply that $a_{1}=0$; then the equation with $i=2$ will imply that $a_{2}=0$; and so on, until we set $a_{d-2}=0$. We cannot ensure $a_{d-1}=0$ because $a_{d-1}$ always appears in those equations multiplied by a different $a_{i}$, instead of squared. Hence we could get a degenerate-linear map.

In case (3), we again look at the action of matrices of the form $[1, t ; 0,1]$. Such matrices map $\left(a_{0} x^{d}+a_{d-1} x y^{d-1}\right) / b_{d} y^{d}$ to

$$
\frac{a_{0} x^{d}+a_{d-1} x y^{d-1}+\left(-a_{0} t^{d}-a_{d-1} t+b_{d} t\right) y^{d}}{b_{d} y^{d}} .
$$

Now the only way a map of the form $\left(a_{0} x^{d}+a_{d-1} x y^{d-1}+a_{d} y^{d}\right) / b_{d} y^{d}$ could degenerate is if the image of the polynomial map $t \mapsto-a_{0} t^{d}-a_{d-1} t+b_{d} t$ misses $a_{d}$, which could only happen if the polynomial were constant, that is, $a_{0}=0$ and $a_{d-1}=b_{d}$, giving us a degenerate-linear map.

Remark 4.7. The importance of the lemma is that in all degenerate cases, the map is necessarily unstable, since $d-1$ (or, in case (2), $d-p^{m}$ ) is always at least as large as $d / 2$.

We can now prove Theorem 4.1 when $n=1$. So if we can always find a $D \subseteq \operatorname{Hom}_{d}^{n, s s}$ that works globally, we can find one over a family in which every map is conjugate to $\left(a_{0} x^{d}+a_{d} y^{d}\right) / b_{d} y^{d}$, or, in characteristic $p$ with $d=p^{m}$,

$$
\frac{a_{0} x^{d}+a_{d-1} x y^{d-1}}{b_{d} y^{d}} \text {. }
$$


It suffices to show that there exists a map with $a_{0}=0$. For this, we use the fixed point polynomial, which is well-defined on this family. If the polynomial is fixed, then all maps in the family may be simultaneously conjugated to the form

$$
\frac{a_{0} x^{d}+a_{d} y^{d}}{b_{d} y^{d}}
$$

(or $\left(a_{0} x^{d}+a_{d-1} x y^{d-1}\right) / b_{d} y^{d}$ ), and then one map must have $a_{0}=0$. If the polynomial varies, then some map will have the point at infinity colliding with another fixed point. This will force the map to be ill-defined at infinity; recall that totally invariant points are simple roots of the fixed point polynomial, unless they are bad. This will force $a_{0}$ to be zero, again.

For higher $n$, the proof is similar. The lemma we need is similar to Lemma 4.6, but is somewhat more complicated:

Lemma 4.8. Let $V$ be the closure of the $\operatorname{PGL}(n+1)$-orbit of the family

$$
\left(c_{0} x_{0}^{d}+b x_{1}^{d}: \varphi_{1}: \ldots: \varphi_{n}\right),
$$

where $\varphi_{i}$ is $x_{j}$-free for all $j<i$.

(1) If the characteristic does not divide $d$, then every $\varphi \in V$ is actually in the $\operatorname{PGL}(n+1)$-orbit of the family, or else it is a degenerate map, whose only possible nonzero coefficients in $\varphi_{0}$ are those without an $x_{0}$ term and those of the form $x_{0} p_{0}$, where there is no nonzero $x_{0}$-term in $p_{0}$.

(2) If the characteristic $p$ satisfies $p \mid d$, with $d \neq p^{m} \| d$, then the same statement as in case (1) holds as long as each $\varphi_{i}$ is in terms of $x_{j}^{p^{m}}$, but with $x_{0} p_{0}$ replaced by $x_{0}^{p^{m}} p_{0}$.

(3) If the characteristic $p$ satisfies $d=p^{m}$, then changing the family to

$$
\left(c_{0} x_{0}^{d}+b x_{0} x_{1}^{d-1}: \varphi_{1}: \ldots: \varphi_{n}\right)
$$

with $\varphi_{i}$ in terms of $x_{j}^{d}$ as in case (2), the same statement as in case (1) holds.

Proof. As in the one-dimensional case, case (2) is reducible to case (1) with $d$ replaced with $d / p^{m}$ and $x_{i}$ with $x_{i}^{p^{m}}$. By Lemma 4.5, we only need to conjugate by upper triangular matrices. Further, we only need to conjugate by matrices of the family $E$, with first row $\left(1, t_{1}, \ldots, t_{n}\right)$ and other rows the same as the identity matrix. This is because we can control the diagonal elements because the condition of being in the family is diagonal matrix-invariant, and we can control the rest by projecting any curve $Z$ of unipotent upper triangular matrices onto $E$.

Set $a_{\boldsymbol{d}}$ to be the $\boldsymbol{x}^{\boldsymbol{d}}$-coefficient in $\varphi_{0}$. For all vectors $\boldsymbol{i}, \boldsymbol{j}, \boldsymbol{k}, \boldsymbol{l}$ with $\boldsymbol{i}+\boldsymbol{j}=\boldsymbol{k}+\boldsymbol{l}$, we have $\left(\begin{array}{l}d \\ i\end{array}\right)\left(\begin{array}{l}d \\ j\end{array}\right) a_{\boldsymbol{i}} a_{\boldsymbol{j}}=\left(\begin{array}{l}d \\ \boldsymbol{k}\end{array}\right)\left(\begin{array}{l}d \\ \boldsymbol{l}\end{array}\right) a_{\boldsymbol{k}} a_{\boldsymbol{l}}$, as long as none of $\boldsymbol{i}, \boldsymbol{j}, \boldsymbol{k}$, or $\boldsymbol{l}$ is in the span of $\boldsymbol{e}_{i}$ 
for $i>0$. Note that $i$ and $i$ are two separate quantities, one an index of coordinates and one an index of monomials.

As in the one-dimensional case, we may set $\boldsymbol{j}=\boldsymbol{i}$ and $\boldsymbol{k}=\boldsymbol{i}-\boldsymbol{e}_{0}+\boldsymbol{e}_{i}$. If $c_{0}=a_{(d, 0, \ldots, 0)} \neq 0$, then by the same argument as before, the values of the $x_{0}^{d-1} x_{i}$ coefficients determine all the rest, and we can conjugate the map back to the desired form. And if $c_{0}=0$, then the value of every coefficient that can occur as $i$ in the above construct is zero; the only coefficients that cannot are those with no $x_{0}$ component and those with a linear $x_{0}$ component.

In case (3), we restrict to matrices of the same form as in case (1), and observe that those matrices only generate extra $x_{i}^{d}$ and $x_{i} x_{1}^{d-1}$ in $\varphi_{0}$. The statement is vacuous if $c_{0}=0$, so assume $c_{0} \neq 0$. For $i=1$, this is identical to the one-dimensional case, so if $c_{0} \neq 0$, then we can find an appropriate $t_{1}$. For higher $i$, if $b \neq 0$, then we can extract $t_{i}$ from the $x_{i} x_{1}^{d-1}$ coefficient, which will necessarily work for the $x_{i}^{d}$ coefficient as well, making the map conjugate to the family; if $b=0$, then the same equations as for $i=1$ hold for higher $i$, and we can again find $t_{i}$ 's conjugating the map to the family.

While we could also control the terms involving a linear (or $p$-power) $x_{0}$ coefficient in the above construction, it is not necessary for our purposes.

To finish the proof of Theorem 4.1, first note that in the closure of the family above, any map for which $c_{0}=0$ is unstable. Indeed, the one-parameter subgroup of PGL $(n+1)$ with diagonal coefficients $t_{0}=n, t_{i}=-1$ for $i>0$, shows instability. Recall that a map is unstable with respect to such a family if $t_{i}>t_{0} d_{0}+\cdots+t_{n} d_{n}$ whenever the $x_{0}^{d_{0}} \ldots x_{n}^{d_{n}}$-coefficient of $\varphi_{i}$ is nonzero. With the above one-parameter subgroup, we have $t_{0} d_{0}+\cdots+t_{n} d_{n}=-d<-1$ for the only nonzero monomials in $\varphi_{i}$ with $i>0$; in $\varphi_{0}$, the maximal value of $t_{0} d_{0}+\cdots+t_{n} d_{n}$ is $t_{0}+t_{i}(d-1)=$ $n-(d-1)<n$.

Now we need to show only that for some map in the family, $c_{0}$ will indeed be zero. So suppose on the contrary that $c_{0}$ is never zero. Then all maps are, after conjugation, in the family $\left(c_{0} x_{0}^{d}+b x_{1}^{d}: \varphi_{1} \ldots: \varphi_{n}\right)$, where the linear subvariety $\varphi_{i}=\varphi_{i+1}=\cdots=\varphi_{n}$ is totally invariant. Now look at the action on the line $x_{2}=\cdots=x_{n}=0$. Every morphism will induce a morphism on this line, so there will be three fixed points on it, counting multiplicity. We now imitate the proof in the one-dimensional case: the totally invariant fixed point on this line, $(1: 0: \ldots: 0)$, will collide with another fixed point, so the map will be ill-defined at it. This means that $(1: 0: \ldots: 0)$ is a bad point, which cannot happen unless $c_{0}=0$.

Trivially, the above theorem for curves shows the same for higher-dimensional families in $\mathrm{M}_{d}^{n, s s}$. An interesting question could be to generalize semistable reduction to higher-dimensional families, for which we may get projective vector bundles just like in the case of curves. Trivially, if we have two proper subvarieties of $\mathrm{M}_{d}^{n, s s}$, 
$V_{1} \subseteq V_{2}$, and a bundle class occurs for $V_{2}$, then its restriction to $V_{1}$ occurs for $V_{1}$. In particular, if we have the trivial class over $V_{2}$, then we also have it over $V_{1}$, as well as any other subvariety of $V_{2}$. This leads to the following question: if the trivial class occurs for every proper closed subvariety of $V_{2}$, does it necessarily occur for $V_{2}$ ? What if we weaken the condition and only require the trivial class to occur for subvarieties that cover $V_{2}$ ?

\section{The trivial bundle case}

For most curves $C \subseteq \mathrm{M}_{d}^{n, s s}$, there occurs a trivial bundle. Since the complement of $\operatorname{Hom}_{d}^{n, s s}$ in $\mathbb{P}^{N}$ has high codimension, this is true by simple dimension counting. Therefore, it is useful to analyze those curves separately, as we have more tools to work with. Specifically, we can use more machinery from geometric invariant theory. We will start by proving Proposition 1.7, restated below:

Proposition 5.1. Let $X$ be a projective variety over an algebraically closed field with an action by a geometrically reductive linear algebraic group $G$. Using the terminology of geometric invariant theory, let $D$ be a complete curve in the stable space $X^{s}$ whose quotient by $G$ is a complete curve $C$; say the map from $D$ to $C$ has degree $m$. Suppose the stabilizer is generically finite, of size $h$, and either $D$ or $C$ is normal. Then there exists a finite subgroup $S_{D} \subseteq G$, of order equal to $m h$, such that for all $x \in D$ and $g \in G, g x \in D$ if and only if $g \in S_{D}$.

Proof. For $x \in D$, we define $S_{D}(x)=\{g \in G: g x \in D\}$. This is a map of sets from an open dense subset of $D$ to $\operatorname{Sym}^{m h}(G)$, and is regular on an open dense subset. We have:

Lemma 5.2. The map from $\operatorname{Sym}^{m h}(G) \times X^{s}$ to $\operatorname{Sym}^{m h}\left(X^{s}\right) \times X^{s}$ defined by sending each $\left(\left\{g_{1}, \ldots, g_{m h}\right\}, x\right)$ to $\left(\left\{g_{1} \cdot x, \ldots, g_{m h} \cdot x\right\}, x\right)$ is proper.

Proof. By standard geometric invariant theory, the map from $G \times X^{s}$ to $X^{s} \times X^{s}$, $(g, x) \mapsto(g \cdot x, x)$, is proper. Thus the map from $G^{m h} \times\left(X^{s}\right)^{m h}$ to $\left(X^{s}\right)^{m h} \times\left(X^{s}\right)^{m h}$ defined by $\left(g_{i}, x_{i}\right) \mapsto\left(g_{i} \cdot x_{i}, x_{i}\right)$ is also proper, as the product of proper maps. Now closed immersions are proper, so the map remains proper if we restrict it to $G^{m h} \times X^{s}$, where we embed $X^{s}$ into $\left(X^{s}\right)^{m h}$ diagonally; the image of this map lands in $\left(X^{s}\right)^{m h} \times X^{s}$. Finally, we quotient out by the symmetric group $S_{m h}$, obtaining

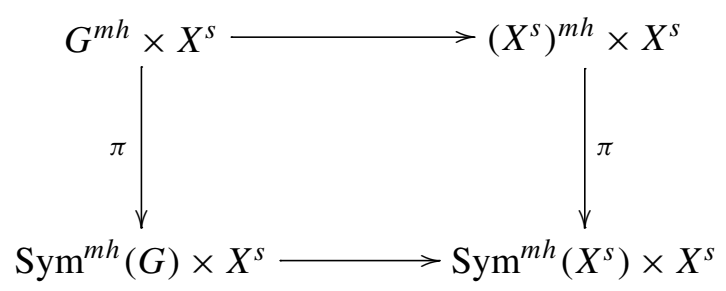


The map on the bottom is already separated and finite-type; we will show it is universally closed. Extend it by some arbitrary scheme $Y$. If

$$
V \subseteq \operatorname{Sym}^{m h}(G) \times X^{s} \times Y
$$

is closed, then so is $\pi^{-1}(V) \subseteq G^{m h} \times X^{s} \times Y$. The map on top is universally closed, so its image is closed in $\left(X^{s}\right)^{m h} \times X^{s} \times Y$. But the map on the right is proper, so the image of $V$ is also closed in $\operatorname{Sym}^{m h}\left(X^{s}\right) \times X^{s} \times Y$.

Now, the rational map $f_{D}(x)=S_{D}(x) \cdot x \in \operatorname{Sym}^{m h}(D)$ can be extended to a morphism on all of $D$, since both $D$ and $\operatorname{Sym}^{m h}(D)$ are proper. This is trivial if $D$ is normal; if it is not normal, but $C$ is normal, then observe that the map factors through $C$ since it is constant on orbits, and then analytically extend it through $C$. But now $\left(f_{D}(x), x\right)$ embeds into $\operatorname{Sym}^{m h}\left(X^{s}\right) \times X^{s}$ as a proper curve. The preimage in $\operatorname{Sym}^{m h}(G) \times X^{s}$ of this curve is also proper; for each $\left(f_{D}(x), x\right)$, it is a finite set of points of the form $(S, x)$ satisfying $S \cdot x=f_{D}(x)$, including $\left(S_{D}(x), x\right)$. Projecting onto the $\operatorname{Sym}^{m h}(G)$ factor, we still get a proper set, which means it must be a finite set of points, as $\operatorname{Sym}^{m h}(G)$ is affine. One of these points will be $S_{D}$, which is then necessarily finite.

Finally, if $g, h \in S_{D}$ and $x \in D$, then $g \cdot h \cdot x \in g \cdot D=D$; therefore $S_{D}$ is a group.

Remark 5.3. The proposition essentially says that the cover $D \rightarrow C$ is necessarily Galois. The generic stabilizer is necessarily a group $H$, normal in $S_{D}$.

Corollary 5.4. With the same notation and conditions as in Proposition 5.1, the map from $D$ to $C$ ramifies precisely at points $x \in D$ such that $\operatorname{Stab}(x)$ intersects $S_{D}$ in a strictly larger group than $H$. Furthermore, the ramification degree is exactly $\left[\operatorname{Stab}(x) \cap S_{D}: H\right]$.

For high $n$ or $d$, the stabilized locus of $\operatorname{Hom}_{d}^{n}$ is of high codimension. Furthermore, most curves in $\operatorname{Hom}_{d}^{n, s s}$ lie in $\operatorname{Hom}_{d}^{n, s}$. Therefore, generically not only is $H$ trivial, but also there are no points on $D$ with nontrivial stabilizer. Thus for most $C$ and $D$, the map $D \rightarrow C$ must be unramified. Thus, when $C$ is rational, generically the degree is 1 .

It's based on this observation that we conjecture the bounds for the nontrivial bundle case in both directions - that is, that if we fix $C$ and the bundle class $\boldsymbol{P}(\mathscr{E})$, then the degree of the map $\pi: D \rightarrow C$ is bounded.

Using the structure result on $\mathrm{M}_{2}^{s s}=\mathbb{P}^{2}$, we can prove much more:

Proposition 5.5. If $C$ is a generic line in $\mathrm{M}_{2}^{s s}$, then it requires a nontrivial bundle. Proof. Generically, $C$ is not the line consisting of the resultant locus, $M_{2}^{s s} \backslash M_{2}$. So it intersects this line at exactly one point. Furthermore, since the resultant Res2 is an $\operatorname{SL}(2)$-invariant section, we have $D \cdot \operatorname{Res}_{2}=m \cdot C$. $\operatorname{Res}_{2}$; we abuse notation 
and use $\operatorname{Res}_{d}^{n}$ to refer to the resultant divisor both upstairs and downstairs. Since the degree of the resultant upstairs is $(n+1) d^{n}=4$ [Jouanolou 1991], we obtain $4 \cdot D .0(1)=m$. In other words, $m \geq 4$.

However, using Proposition 5.1, we will show $m \leq 2$ generically. The generic stabilizer is trivial, and the stabilized locus is a cuspidal cubic in $\mathbb{P}^{2}$, on which the stabilizer is isomorphic to $\mathbb{Z} / 2 \mathbb{Z}$, except at the cusp, where it is $S_{3}$. The generic line $C$ will intersect this cuspidal curve at three points, none of which is the cusp. Therefore, $h=1$, and there are at most three points of ramification, with ramification degree 2. By Riemann-Hurwitz, the maximum $m$ is 2 , contradicting $m \geq 4$.

\section{Acknowledgements}

The author would like to thank his Ph.D. advisor Shouwu Zhang for proposing this problem, Johan de Jong for many helpful discussions about Section 5, and the anonymous reviewer for fixing the presentation and proof of Theorem 1.4.

\section{References}

[Jouanolou 1991] J.-P. Jouanolou, "Le formalisme du résultant", Adv. Math. 90:2 (1991), 117-263. MR 93g:14003 Zbl 0747.13007

[Levy 2011] A. Levy, "The space of morphisms on projective space”, Acta Arith. 146:1 (2011), 13-31. MR 2012d:37211 Zbl 05834866

[Milnor 2006] J. Milnor, Dynamics in one complex variable, 3rd ed., Annals of Mathematics Studies 160, Princeton University Press, 2006. MR 2006g:37070 Zbl 1085.30002

[Mumford and Fogarty 1982] D. Mumford and J. Fogarty, Geometric invariant theory, 2nd ed., Ergeb. Math. Grenzgeb. 34, Springer, Berlin, 1982. MR 86a:14006 Zbl 0504.14008

[Petsche et al. 2009] C. Petsche, L. Szpiro, and M. Tepper, "Isotriviality is equivalent to potential good reduction for endomorphisms of $\mathbb{P}^{N}$ over function fields", J. Algebra 322:9 (2009), 3345-3365. MR 2011e:14023 Zbl 1190.14013

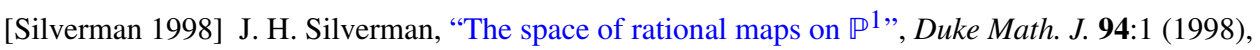
41-77. MR 2000m:14010 Zbl 0966.14031

[Silverman 2007] J. H. Silverman, The arithmetic of dynamical systems, Graduate Texts in Mathematics 241, Springer, New York, 2007. MR 2008c:11002 Zbl 1130.37001

[Szpiro et al. 2010] L. Szpiro, M. Tepper, and P. Williams, "Resultant and conductor of geometrically semi-stable self maps of the projective line over a function field", preprint, 2010. arXiv 1010.5030v1 [Zhang 1996] S. Zhang, "Heights and reductions of semi-stable varieties", Compositio Math. 104:1 (1996), 77-105. MR 97m:14027 Zbl 0924.11055

Communicated by Bjorn Poonen

Received 2011-06-15 Revised 2011-08-02 Accepted 2011-09-11

levy@math.columbia.edu Department of Mathematics, Brown University,

Providence, RI 02912, United States

Department of Mathematics, Columbia University,

New York, NY 10027, United States 


\section{Algebra \& Number Theory}

msp.berkeley.edu/ant

\section{EDITORS}

MANAGING EDITOR

Bjorn Poonen

Massachusetts Institute of Technology

Cambridge, USA

\author{
EDITORIAL BOARD CHAIR \\ David Eisenbud \\ University of California \\ Berkeley, USA
}

\section{BOARD OF EDITORS}

Georgia Benkart

Dave Benson

Richard E. Borcherds

John H. Coates

J-L. Colliot-Thélène

Brian D. Conrad

Hélène Esnault

Hubert Flenner

Edward Frenkel

Andrew Granville

Joseph Gubeladze

Ehud Hrushovski

Craig Huneke

Mikhail Kapranov

Yujiro Kawamata

János Kollár

Yuri Manin

Barry Mazur

Philippe Michel
University of Wisconsin, Madison, USA

University of Aberdeen, Scotland

University of California, Berkeley, USA

University of Cambridge, UK

CNRS, Université Paris-Sud, France

University of Michigan, USA

Freie Universität Berlin, Germany

Ruhr-Universität, Germany

University of California, Berkeley, USA

Université de Montréal, Canada

San Francisco State University, USA

Hebrew University, Israel

University of Virginia, USA

Yale University, USA

University of Tokyo, Japan

Princeton University, USA

Northwestern University, USA

Harvard University, USA

École Polytechnique Fédérale de Lausanne
Susan Montgomery

Shigefumi Mori

Raman Parimala

Jonathan Pila

Victor Reiner

Karl Rubin

Peter Sarnak

Joseph H. Silverman

Michael Singer

Vasudevan Srinivas

J. Toby Stafford

Bernd Sturmfels

Richard Taylor

Ravi Vakil

Michel van den Bergh

Marie-France Vignéras

Kei-Ichi Watanabe

Andrei Zelevinsky

Efim Zelmanov
University of Southern California, USA

RIMS, Kyoto University, Japan

Emory University, USA

University of Oxford, UK

University of Minnesota, USA

University of California, Irvine, USA

Princeton University, USA

Brown University, USA

North Carolina State University, USA

Tata Inst. of Fund. Research, India

University of Michigan, USA

University of California, Berkeley, USA

Harvard University, USA

Stanford University, USA

Hasselt University, Belgium

Université Paris VII, France

Nihon University, Japan

Northeastern University, USA

University of California, San Diego, USA

\section{PRODUCTION}

production@msp.org

Silvio Levy, Scientific Editor

See inside back cover or www.jant.org for submission instructions.

The subscription price for 2012 is US \$175/year for the electronic version, and \$275/year ( $\$ 40$ shipping outside the US) for print and electronic. Subscriptions, requests for back issues from the last three years and changes of subscribers address should be sent to Mathematical Sciences Publishers, Department of Mathematics, University of California, Berkeley, CA 94720-3840, USA.

Algebra \& Number Theory (ISSN 1937-0652) at Mathematical Sciences Publishers, Department of Mathematics, University of California, Berkeley, CA 94720-3840 is published continuously online. Periodical rate postage paid at Berkeley, CA 94704, and additional mailing offices.

ANT peer review and production are managed by EditFLOW ${ }^{\circledR}$ from Mathematical Sciences Publishers.

PUBLISHED BY

mathematical sciences publishers

http://msp.org/

A NON-PROFIT CORPORATION

Typeset in IATEX

Copyright ( 2012 by Mathematical Sciences Publishers 


\section{Algebra \& Number Theory}

\section{Volume $6 \quad$ No. $7 \quad 2012$}

On the rank of the fibers of rational elliptic surfaces

Cecília Salgado

Néron's pairing and relative algebraic equivalence

CÉDRIC PÉPIN

Free subalgebras of quotient rings of Ore extensions

JASON P. BELL and DANIEL ROGALSKI

Classes de cycles motiviques étales

1369

BRUNO KAHN

Higher-order Maass forms

1409

ROELOF BRUGGEMAN and NiKOLAOS DIAMANTIS

Log canonical thresholds, $F$-pure thresholds, and nonstandard extensions

Bhargav Bhatt, Daniel J. Hernández, Lance Edward Miller and Mircea MUSTAȚĂ

The semistable reduction problem for the space of morphisms on $\mathbb{P}^{n}$

ALON LEVY

Grothendieck's trace map for arithmetic surfaces via residues and higher adèles

MATTHEW MORROW

Crystalline extensions and the weight part of Serre's conjecture

Toby GeE, Tong LiU and DAVID SAVITT

Annihilating the cohomology of group schemes 